15

\title{
Особенности измерения поверхностного распределения электрического потенциала локальным зондом на основе полевого транзистора с каналом-нанопроводом
}

\author{
(C) И.В. Божьев, ${ }^{1,2}$ В.А. Крупенин, ${ }^{1}$ Д.Е. Преснов, ${ }^{1,3}$ И.И. Циняйкин, ${ }^{1,2}$ А.А. Дорофреев, ${ }^{1,2}$ А.С. Трифонов ${ }^{1,2}$ \\ ${ }^{1}$ Центр квантовых технологий, Московский государственный университет им. М.В. Ломоносова, \\ 119991 Москва, Россия \\ ${ }^{2}$ Физический факультет, Московский государственный университет им. М.В. Ломоносова, \\ 119991 Москва, Россия \\ ${ }^{3}$ Научно-исследовательский институт ядерной фризики имени Д.В. Скобельцына, Московский государственный \\ университет им. М.В. Ломоносова, \\ 119991 Москва, Россия \\ e-mail: bozhjev.ivan@physics.msu.ru, trifonov.artem@physics.msu.ru
}

Поступило в Редакцию 17 октября 2019г.

В окончательной редакции 13 ноября 2019г.

Принята к публикации 29 ноября 2019г.

\begin{abstract}
Продемонстрирован неразрушающий метод сканирующей зондовой микроскопии для одновременного измерения топографии поверхности и распределения электрического поля (заряда, потенциала). Сканирование поверхности осуществлено с помощью зондового датчика на основе камертона: взаимодействие с поверхностью осуществляется острым краем кремниевого чипа, закрепленного на одной из ножек кварцевого резонатора (камертона). Детектирование электрических потенциалов происходило с помощью полевого транзистора с каналом нанопроводом, сформированного вблизи острого края кремниевого чипа. Из-за невысокой добротности колебательной системы сканирование стандартными алгоритмами движения зонда над поверхностью приводили к быстрому износу и даже разрушению зонда. Был использован оригинальный алгоритм сканирования, основанный на алгоритме поточечного измерения рельефа поверхности и минимизирующий время взаимодействия зонда и исследуемого объекта. При этом минимальное время нахождения зонда в каждой точке поверхности составляло $1.0-1.6 \mathrm{~ms}$ и определялось временем отклика полевого транзистора на изменение электрического поля (время измерения одного кадра составило 20-30 min). Пространственное разрешение метода составило $10 \mathrm{~nm}$ для топографии и $20 \mathrm{~nm}$ для полевого профиля образца при одновременном их измерении. Полевое разрешение изготовленных чипов находилось в диапазоне $2-5 \mathrm{mV}$ и определялось чувствительностью нанопровода полевого транзистора и расстоянием от нанопровода до вершины зонда.
\end{abstract}

Ключевые слова: сканирующая зондовая микроскопия, полевой транзистор с каналом-нанопроводом, локальный зарядовый/полевой сенсор, кремний на изоляторе, зарядовая чувствительность.

DOI: $10.21883 /$ JTF.2020.05.49192.342a-19

\section{Введение}

Для проведения многих исследований в микроэлектронике, биологии, химии и медицине необходим высокочувствительный неразрушающий анализ пространственного распределения профиля электрического потенциала с высоким пространственным и полевым разрешением. К настоящему времени известны десятки методик сканирующей зондовой микроскопии (СЗМ) для измерения электрических свойств твердотельных или мягких поверхностей [1-14]. Электростатическая силовая микроскопия (EFM) и силовая микроскопия зонда Кельвина (KFM) основаны на эффекте электростатического взаимодействия между смещенным зондом и образцом для расчета количественного значения электрического потенциала. Эти методы демонстрируют разрешение по потенциалу в несколько милливольт при низких температурах [2]. Сканирующая емкостная микроскопия использует только электрические измерения $[3,4]$ и имеет тот же уровень разрешения. Также имеются работы [5-11], в которых на вершине зонда СЗМ были изготовлены активные датчики: резистивный датчик [5], полевые транзисторы [6-8], одноэлектронные транзисторы [9-10] и наноэлектромеханические устройства [11]. Перспективным кандидатом на использование локального датчика или датчика для СЗМ является полевой транзистор, у которого канал-нанопровод модифицирован специальными молекулами-ключами. В основном такие транзисторы широко используются при разработке высокочувствительных биосенсоров для обнаружения чрезвычайно низких концентраций специфических молекул (белков, ДНК, РНК) в биологических системах [15-21]. Среди всех устройств наиболее чувствительным полевым/зарядовым сенсором является одноэлектронный транзистор, работающий только при очень низких температурах [22]. При уменьшении разме- 
ра канала-нанопровода до 20 - $30 \mathrm{~nm}$ одноэлектронный транспорт в нем реализуется через одиночные примесные атомы [23-25]. Полевые транзисторы работают в широком диапазоне температур от миликельвин до комнатной температуры. Чувствительность к заряду сканирующего устройства на основе полевого транзистора при комнатной температуре начинается с 100 электронов [26] и в настоящее время достигает уже десятков электронов [7,8,27]. Теоретически чувствительность к заряду полевого транзистора с каналом-нанопроводом на несколько порядков меньше, чем заряд одиночного электрона $[28,29]$.

Авторами ранее был продемонстрирован прототип зондового устройства [30,31] для СЗМ, представляющий собой чип, на котором изготовлен транзистор с каналомнанопроводом, находящийся менее чем в $20 \mathrm{~nm}$ от угла чипа. Острие чипа с транзистором используется как зондовый датчик силового взаимодействия с поверхностью исследуемого образца, а транзистор с каналомнанопроводом как датчик электрического поля вблизи поверхности образца: ток через полевой транзистор с каналом-нанопроводом зависит от внешнего электрического поля, источником которого является исследуемый образец. Таким образом, ток через полевой транзистор пропорционален величине электрического поля (электрического потенциала, заряда) образца. Было достигнуто разрешение профиля потенциала исследуемой поверхности лучше, чем $10 \mathrm{mV}$ в полосе $0.1-100 \mathrm{~Hz}$.

$\mathrm{B}$ настоящей работе описаны особенности алгоритма сканирования и определены значения пространственного и полевого разрешения.

\section{1. Зондовый датчик силового взаимодействия на основе камертона}

Для сканирования поверхности использовался сканирующий зондовый микроскоп SmartSPM-1000 производства компании Aist-NT (Зеленоград, Россия). Сканирование осуществлялось с помощью кварцевого резонатора, имеющего форму камертона. На одну из ножек камертона приклеивался чип с полевым транзистором (рис. $1, a)$, расположенным вблизи края чипа (рис. $1, b)$. При этом силовое взаимодействие с поверхностью реализуется тем кончиком чипа, рядом с которым расположен полевой транзистор с каналом нанопроводом. В нашем случае ножки камертона расположены параллельно поверхности исследуемого образца, т.е. используется режим нормальных сил. Высокая жесткость камертонов по сравнению с обычными зондами для СЗМ позволяет работать с очень малыми амплитудами колебаний $($ менее $1 \mathrm{~nm})$. При возбуждении в камертоне вынужденных механических колебаний генерируется переменное электрическое напряжение, повторяющее характер механических колебаний. Раскачка осуществляется на резонансной частоте камертона. При взаимодействии с

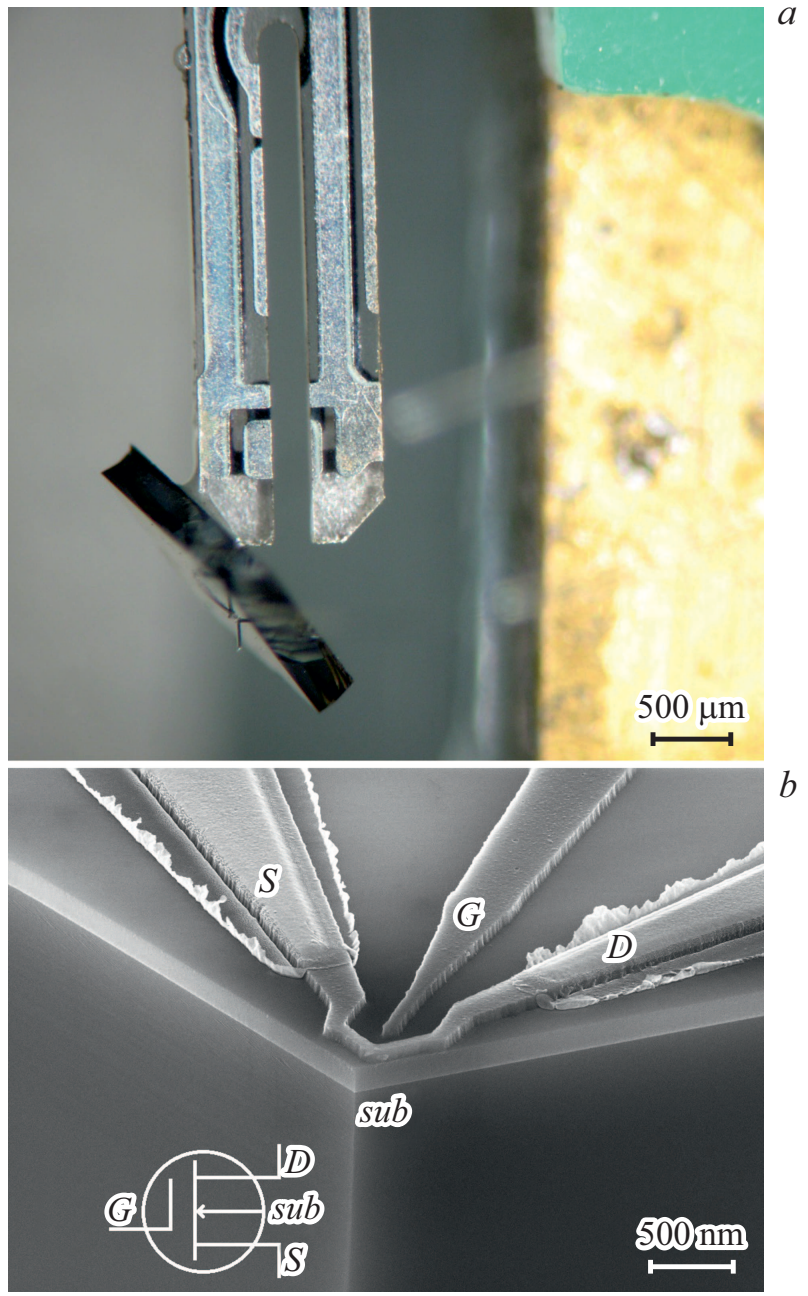

Рис. 1. $a-$ ножки кварцевого резонатора с приклеенным измеряющим чипом; $b-$ изображение в растровом электронном микроскопе (РЭМ) края чипа с полевым транзистором. На вставке схема полевого транзистора с изолированным затвором. Элементы транзистора: $D-$ сток, $S-$ исток, $G-$ затвор и $s u b-$ подложка обозначены также на изображении РЭМ.

поверхностью характер колебаний камертона меняется, что приводит соответственно к изменению параметров генерируемого напряжения (амплитуды, фазы и частоты). Эти параметры используются в качестве сигналов обратной связи.

\section{2. Резонансные характеристики, добротности}

Основными количественными характеристиками кварцевого резонатора являются резонансная частота колебаний и добротность. Все используемые кварцевые резонаторы имели резонансную частоту $32768 \mathrm{kHz}$ и добротность от 10000 до 15000 (в вакуумированном корпусе). После извлечения резонатора из корпуса его 

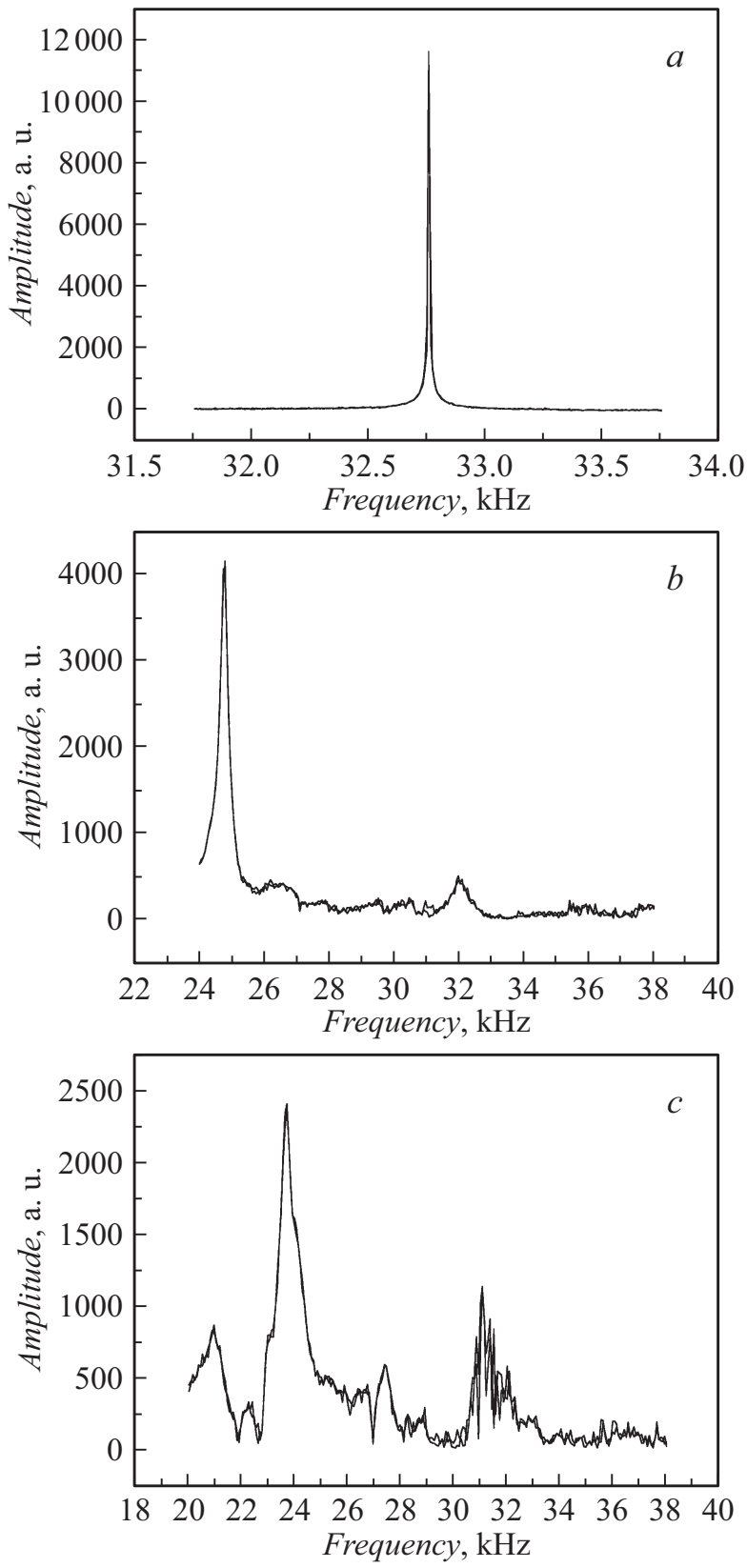

Рис. 2. $a-$ амплитудно-частотная характеристика кварцевого резонатора, извлеченного из корпуса и приклеенного к измерительной плате. Добротность - 4096, резонансная частота $-32.7 \mathrm{kHz} ; b-$ амплитудно-частотная характеристика кварцевого резонатора, на одной ножке которого приклеен кремниевый чип. Добротность - 147, основная резонансная частота $-24.7 \mathrm{kHz} ; c-$ амплитудно-частотная характеристика кварцевого резонатора, на одной ножке которого приклеен кремниевый чип. Добротность - 67, основная резонансная частота $-24.7 \mathrm{kHz}$. Присутствуют еще 3 резонанса -20.9 , 27.5 и $31 \mathrm{kHz}$.

резонансная частота практически не менялась, а добротность падала до величины $4100 \pm 100$ (рис. 2 a). Масса каждой ножки кварцевого резонатора $\sim 1.3 \mathrm{mg}$. Масса чипа сравнима с этой величиной $\sim 0.8 \mathrm{mg}$ при размерах чипа $0.3 \times 1.0 \times 1.0 \mathrm{~mm}^{3}$, поэтому приклеивание чипа с полевым транзистором на кварцевый резонатор существенно меняет добротность и резонансную частоту всей системы за счет появления сильной асимметрии масс в ножках кварцевого резонатора. Эта асимметрия приводила к сдвигу резонансной частоты колебаний кварцевого резонатора в область низких частот на $10-30 \%$ (рис. $2, b$ ). В среднем добротность зонда (кварцевый резонатор с чипом) составляла $150 \pm 30$, таким образом, закрепленный на кварцевом резонаторе чип уменьшал добротность в $\sim 25$ раз. Максимальная наблюдаемая добротность составляла 360 . В случае не симметричного приклеивания чипа на ножку кварцевого резонатора на амплитудно-частотной характеристике появлялись несколько резонансных пиков (рис. 2, $c$ ).

\section{3. Особенности сканирования}

Относительно невысокая добротность камертона приводит к неточностям в работе обратной связи, поддерживающей постоянной высоту зонда над измеряемой поверхностью. При этом возможны механические повреждения („стачивание“ и сколы) вершины зонда измеряющего чипа. Пример таких повреждений измеряющего чипа показан на рис. 3. Для минимизации времени взаимодействия зонда с поверхностью был реализован алгоритм сканирования, основанный на поточечном измерении рельефа поверхности (jumping mode) [32,33], в котором при перемещении между измеряемыми точками поверхности зонд перемещается на большом расстоянии от поверхности. В каждой точке сканирования происходит подвод зонда до достижения определенного уровня взаимодействия с поверхностью, после чего сразу же, либо с заданной временно́й задержкой (необходимой для регистрации отклика полевого сенсора) выполняется отвод зонда от поверхности и перемещение к следующей точке. Подъем осуществляется на фиксированную высоту (а не до достижения определенного уровня амплитуды колебаний). Между точками сканирования движение происходит фактически без обычной обратной связи. При отводе камертона от поверхности и при передвижении между точками необходимо учитывать общий наклон поверхности образца и плоскости сканирования. Этот угол определяется перед каждым сканированием во время пробных подводов камертона к поверхности. Для определения оптимальных параметров работы алгоритма регистрировались все параметры образца во время подвода/отвода камертона. Реализованный алгоритм сканирования после настройки позволял проводить полностью неразрушающие измерения поверхностей с размерами поля сканирования до $100 \times 100 \mu \mathrm{m}$ и рельефом поверхности до $300 \mathrm{~nm}$. Достигнутое максимальное время сканирования одним чипом без ухудшения качества получаемой картинки за счет износа вершины зонда составляло $40 \mathrm{~h}$ (порядка 100 кадров). Общее время 

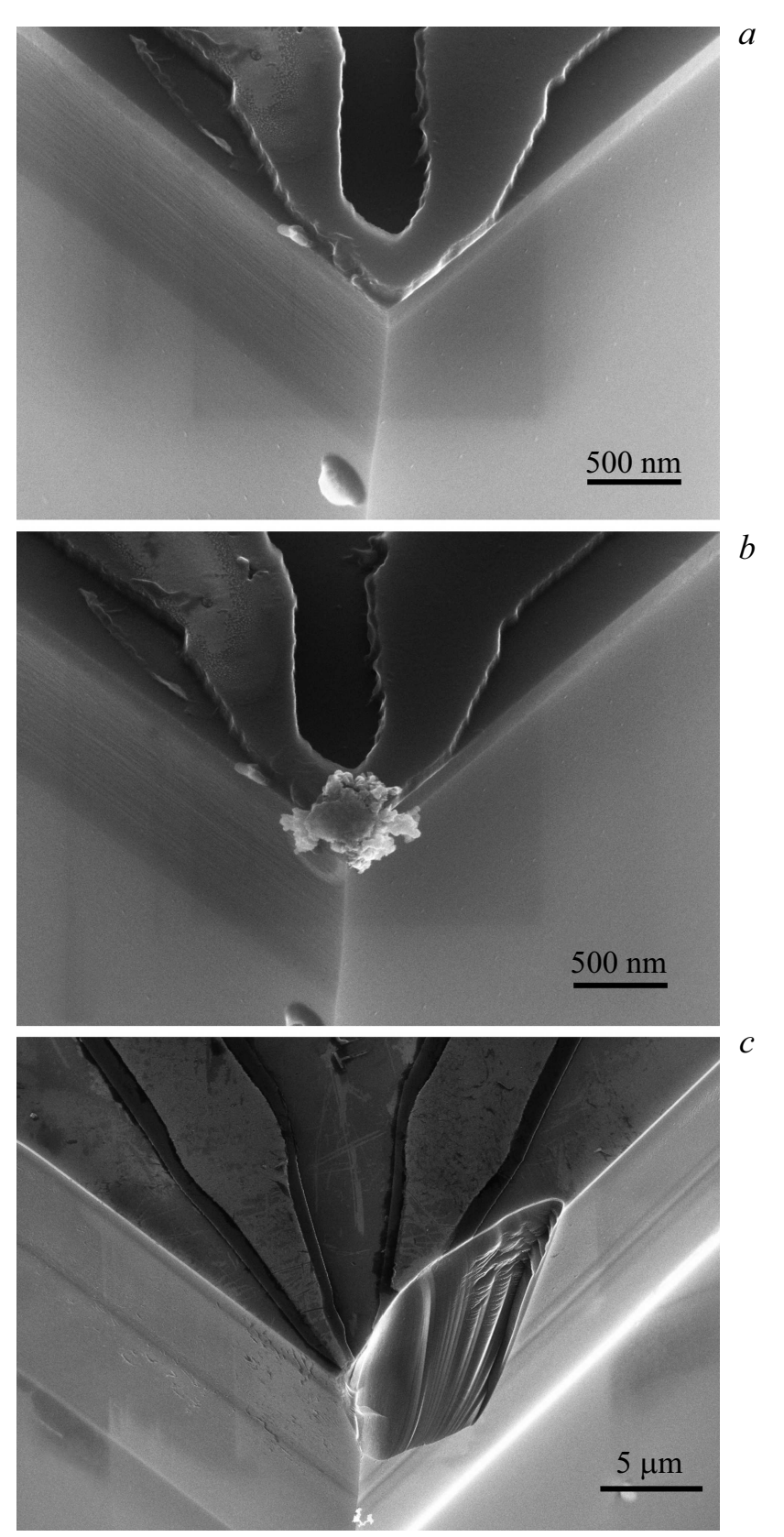

Рис. 3. Примеры механических повреждений вершины измерительного зонда: $a$-чип перед измерениями; $b-$ чип, которым сканировали в течение $30 \mathrm{~min} ; c$ - дополнительное сканирование в течение еще $60 \mathrm{~min}$, после которого сенсор перестал работать. Величина „стачивания“ вершины зонда после $30 \mathrm{~min}$ сканирования составила величину $\sim 300 \mathrm{~nm}$, а после 90 min откололся край чипа размером $\sim 8 \mu \mathrm{m}$.

сканирования одного кадра $(256 \times 256$ точек $)$ составляло $20-30 \mathrm{~min}$.

\section{4. Пространственное и полевое разрешение}

Для демонстрации работы сенсора были изготовлены структуры, представляющие собой металлические по-
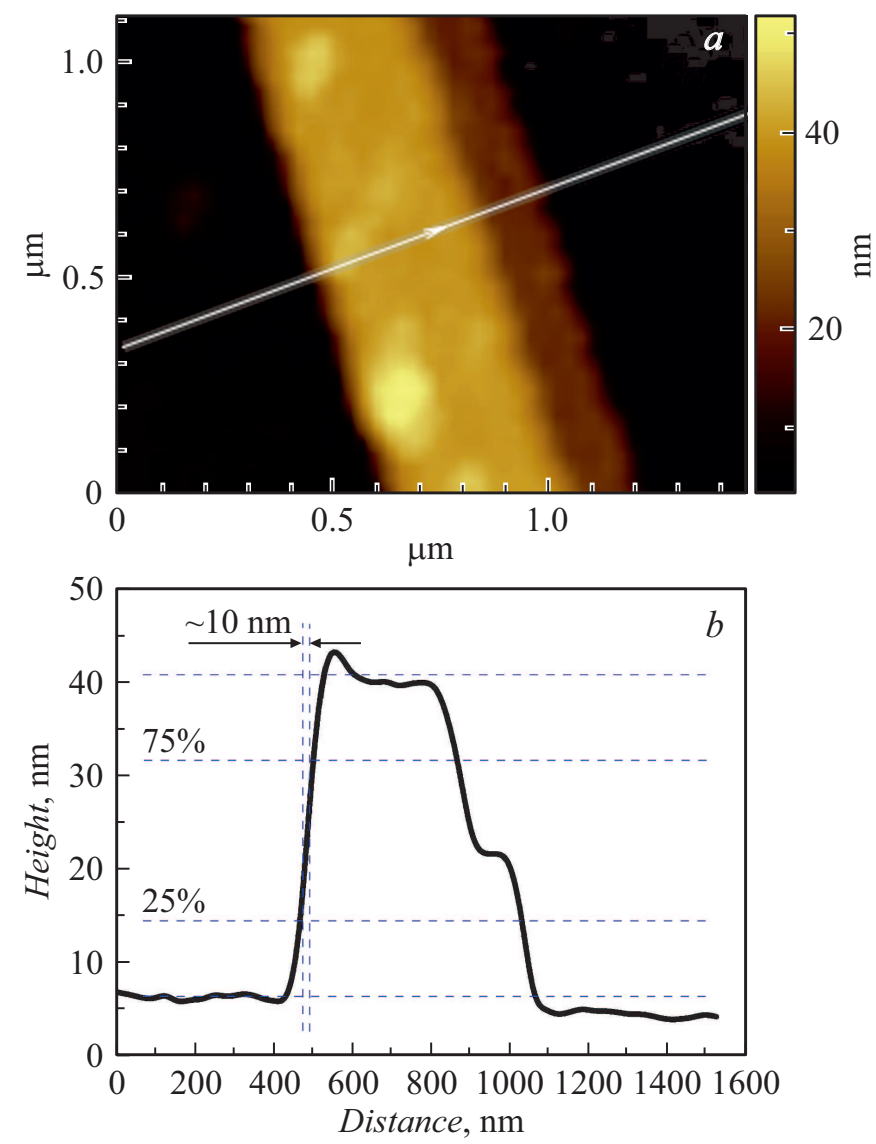

Рис. 4. Топография $(a)$ и сечение $(b)$ тестовой структуры, измеренные камертоном с чипом (резонансная частота $24.8 \mathrm{kHz}$, добротность - 190). Пространственное разрешение при измерении топографии $\sim 10 \mu \mathrm{m}$.

лоски (ширина от 100 до $1000 \mathrm{~nm}$ ) на кремниевом чипе, на которые подавалось постоянное напряжение от -7 до +7V [34]. В эксперименте пространственное разрешение определялось как горизонтальное расстояние между вертикальными отсечками, проведенными на высоте 25 и 75\% характерной ступени на изображении (рис. 4, $b$, $5, b)[35]$.

Пространственное разрешение при измерении топографии. Пространственное разрешение при измерении топографии системы зависело от радиуса закругления вершины зонда $(5-15 \mathrm{~nm})$ и добротности колебательной системы (120-360). Латеральное разрешение для чипов с добротностью 150-300 было порядка $10 \mathrm{~nm}$, вертикальное разрешение $-0.5 \mathrm{~nm}$ (рис. 4). У чипов с меньшей добротностью 75-150 и латеральное и вертикальное разрешения были в 3 раза хуже.

Пространственное разрешение отклика сенсора. Пространственное разрешение сигнала полевого сенсора с высокой добротностью было $\sim 20 \mathrm{~nm}$ (рис. 5), что в два раза хуже пространственного разрешения для топографии. Это связано с тем, что детектируемые объекты (металлические полоски) получаются размытыми по сравнению с топографией из-за того, что нанопровод 

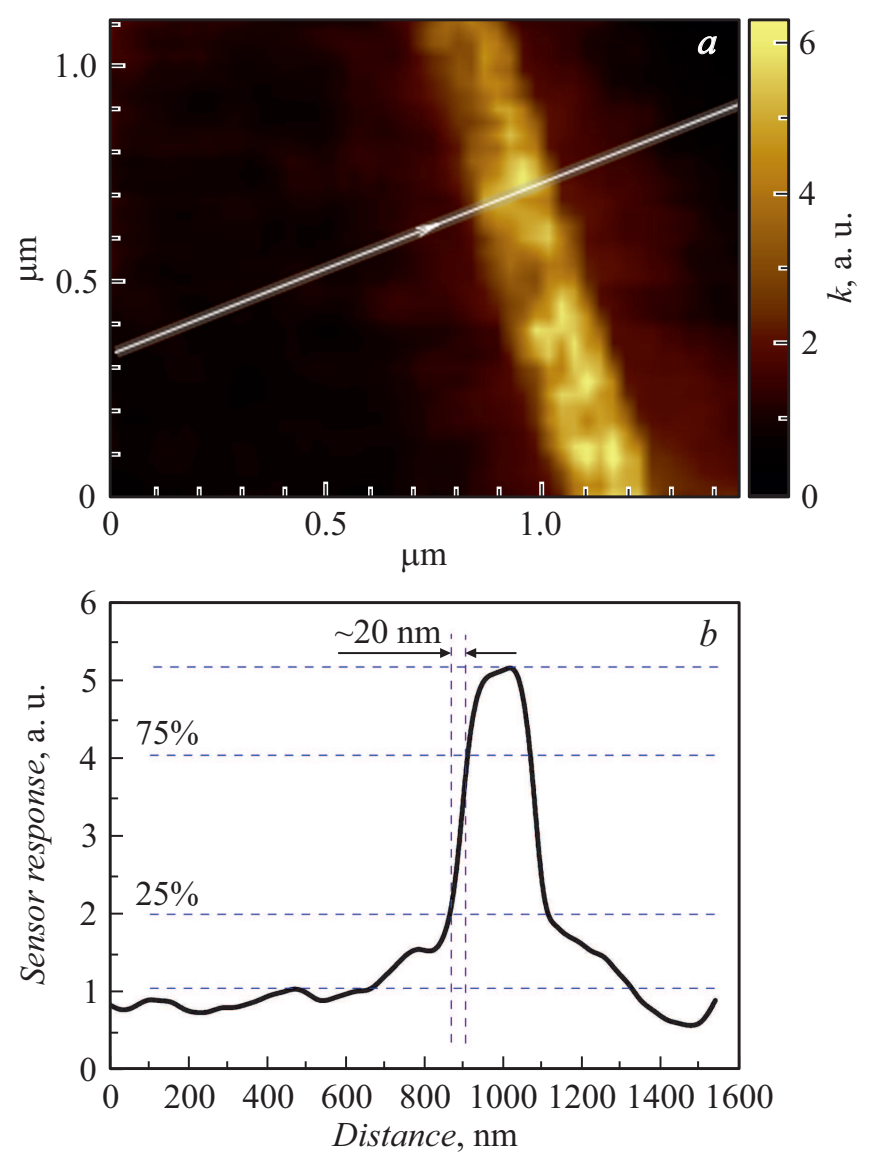

Рис. 5. Карта отклика сенсора $(a)$ и его сечение $(b)$, измеренное камертоном с чипом (резонансная частота $-24.8 \mathrm{kHz}$, добротность - 190). Пространственное разрешение при измерении полевого отклика сенсора $\sim 20 \mathrm{~nm}$. Одновременно измеренная топография приведена на рис. $4, a$.

чувствует поле от электрода не только находясь над ним, но и на некотором расстоянии сбоку.

Полевое разрешение отклика сенсора. Полевое разрешение сигнала отклика сенсора во время сканирования определяется полевой чувствительностью нанопровода (наклоном его сигнальной характеристики), токовым шумом в транзисторе, шумами регистрирующей электроники и расстоянием от нанопровода до исследуемого объекта и его геометрией. Порог полевой чувствительности транзисторов определяется их собственным шумом. Его величина в эксперементе варьировалась в диапазоне от 2 до $5 \mathrm{mV} / \sqrt{\mathrm{Hz}}$ по отношению к собственному затвору транзистора. Шум измерительной электроники составлял величину $\sim 100 \mu \mathrm{V} / \sqrt{\mathrm{Hz}}$. Расстояние между вершиной сканирующего измерительного зонда и поверхностью образца в момент измерения сигнала находилось в диапазоне нескольких нанометров. Расстояние от нанопровода до исследуемого объекта определялось глубиной залегания объекта в образце $\left(d_{1}\right)$ и расстоянием от нанопровода до вершины зонда $\left(d_{2}\right)$. В тестовом образце проводящие электроды были на поверхности $\left(d_{1}=0\right)$. Расстояние $d_{2}$ варьировалось для
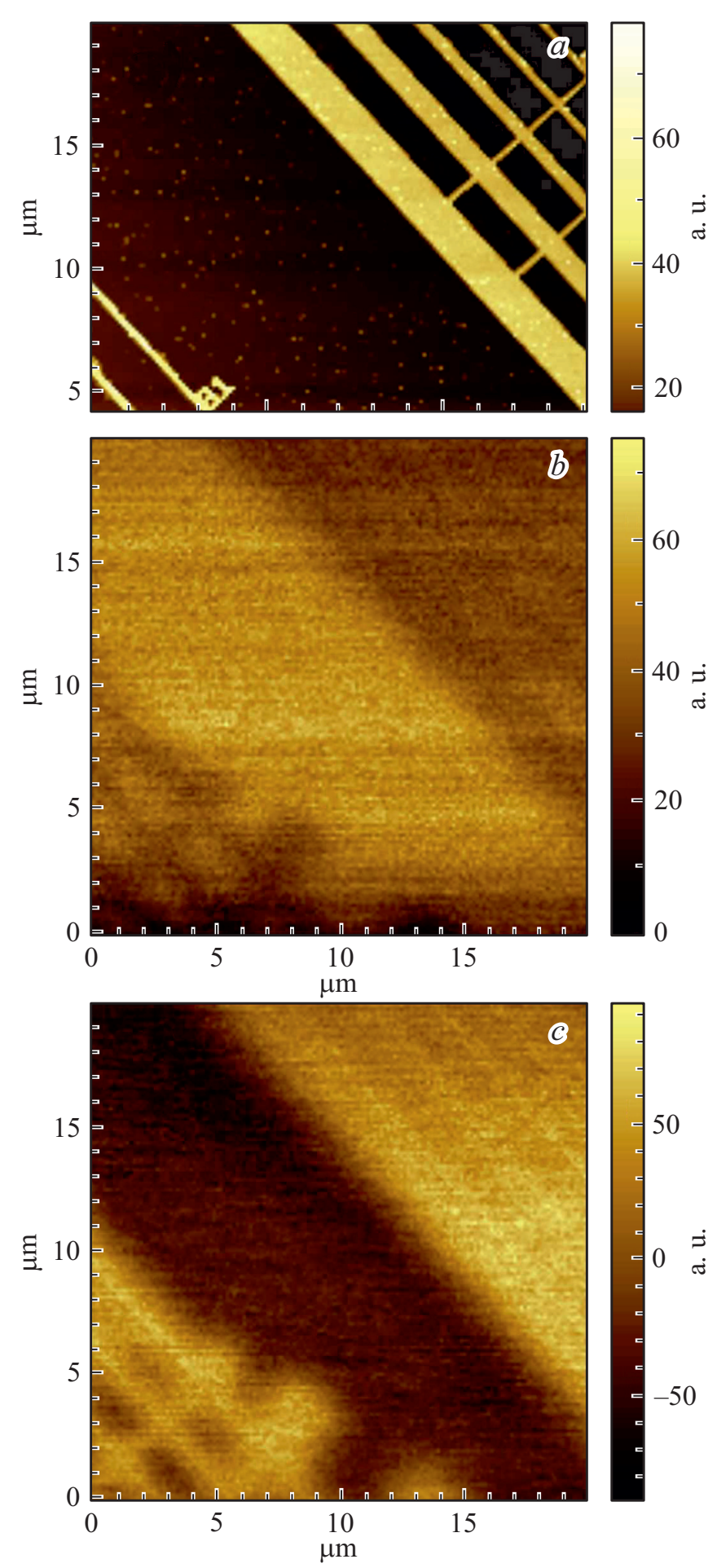

Рис. 6. Топография $(a)$ и зависимости контраста полевого профиля образца от величины задержки измерения отклика сенсора $t$ : (b) $t=0.4 \mathrm{mc},(c) t=1.6 \mathrm{~ms}$.

различных чипов от 300 до $0 \mathrm{~nm}$. Для чипов, на которых полевой транзистор располагался близко к вершине зонда $\left(d_{2}<50 \mathrm{~nm}\right)$, полевое разрешение составляло величину 2-5 mV. Как было показано в [29], предельная чувствительность таких транзисторов на 2 порядке ниже. 
Время отклика нанопровода. Время отклика сенсора $\tau$ определялось в основном емкостями подводящих электродов и внутренним сопротивлением полевого транзистора в рабочей точке. Взаимная емкость нанопровода и исследуемого объекта на несколько порядков меньше. Для оценки величины измерялись семейства кривых подвода/отвода зонда к/от поверхности образца при разных скоростях движения зонда. При подводе зонда к поверхности ток через нанопровод изменялся быстрее, чем при отводе. Оценка характерного времени отклика транзистора производилась следующим образом: считалась разность наклона при подводе и отводе на участках вблизи образца. Далее считалось, что время изменения разницы наклонов от 10 до 90\% равны $3 \tau$. Для изготовленных сенсоров $\tau$ оказалось в диапазоне $0.4-0.8 \mathrm{~ms}$, поэтому при измерении отклика транзистора необходимо устанавливать время задержки перед измерением тока $(t)$ через нанопровод как минимум в 3 раза больше, чем $\tau$. На рис. 6 представлены топография и токовые изображения, иллюстрирующие зависимость контраста от величины задержки.

\section{Заключение}

Продемонстрирован метод сканирующей зондовой микроскопии, в котором сканирование поверхности осуществлялось острой вершиной кремниевого чипа (зонда), закрепленного на одной из ножек кварцевого резонатора. При этом вблизи вершины зонда формировался полевой транзистор с каналом-нанопроводом, который является сенсором электрического поля. Таким образом, метод позволяет измерять одновременно топографию и распределение электрического поля (заряда, потенциала) исследуемого образца. Из-за невысокой добротности колебательной системы сканирование стандартными алгоритмами движения зонда над поверхностью приводили к быстрому износу и даже разрушению зонда. Был реализован алгоритм сканирования, минимизирующий время взаимодействия зонда и исследуемого объекта. Минимальное время нахождения зонда в каждой точке поверхности составило $1.0-1.6 \mathrm{~ms}$ и определялось временем отклика полевого транзистора на изменение электрического поля. Пространственное разрешение метода составило $\sim 10$ и $\sim 20 \mathrm{~nm}$ при одновременном измерении топографии и полевого профиля образца, соответственно. Полевое разрешение изготовленных чипов находилось в диапазоне $2-5 \mathrm{mV}$ и определялось чувствительностью нанопровода полевого транзистора и расстоянием от нанопровода до вершины зонда. К преимуществам метода следует отнести высокую достигнутую полевую чувствительность, которая может быть улучшена на два порядка за счет совершенствования технологии изготовления сенсора (полевого транзистора) и оптимизации алгоритмов сканирования. Проблема большего времени сканирования, чем у аналогичных методик С3М (например, метода зонда Кельвина), может быть преодолена разработкой согласующей электроники, приближенной к измерительному транзистору.

\section{Финансирование работы}

Работа выполнена при финансовой поддержке Российского научного фонда (РНФ грант 16-12-00072).

\section{Благодарности}

Божьев И.В. выражает отдельную благодарность фонду развития теоретической физики и математики „БАЗИС“. В работе использовалось оборудование Учебнометодического центра литографии и микроскопии МГУ им. М.В. Ломоносова.

\section{Конфликт интересов}

Авторы заявляют, что у них нет конфликта интересов.

\section{Список литературы}

[1] Nonnenmacher M., O'Boyle M.P., Wickramasinghe H.K. // Appl. Phys. Lett. 1991. Vol. 58. N 25. P. 2921-2923. https://doi.org/10.1063/1.105227

[2] Ligowski M., Moraru D., Anwar M., Mizuno T., Jablonski R., Tabe M. // Appl. Phys. Lett. 2008. Vol. 93. N 14. P. 142101. https://doi.org/10.1063/1.2992202

[3] Williams C.C., Hough W.P., Rishton S.A. // Appl. Phys. Lett. 1989. Vol. 55. N 2. P. 203-205. https://doi.org/10.1063/1.102096

[4] Matey J.R., Blanc J. // Appl. Phys. Lett. 1985. Vol. 57. N 5. P.1437-1444. https://doi.org/10.1063/1.334506

[5] Park H., Jung J., Min D.K., Kim S., Hong S. // Appl. Phys. Lett. 2004 Vol. 84. N 10. P. 1734-1736. https://doi.org/10.1063/1.1667266

[6] Lee S.H., Lim G., Moon W., Shin H., Kim C.-W. // Ultramicroscopy. 2008. Vol. 108. N 10. P. 1094-1100. https://doi.org/10.1016/j.ultramic.2008.04.034

[7] Shin K., Kang D.S., Lee S.H., Moon W. // Ultramicroscopy. 2015. Vol. 159. N 1. P. $1-10$. https://doi.org/10.1016/j.ultramic.2015.07.007

[8] Ko H., Ryu K., Park H., Park C., Jeon D., Kim Y.K., Jung J., Min D.-K., Kim Y., Lee H.N., Park Y., Shin H., Hong S. // Nano Lett. 2011. Vol. 11. N 4. P. 1428-1433. https://doi.org/10.1021/n1103372a

[9] Brenning H.T.A., Kubatkin S.E., Erts D., Kafanov S.G., Bauch T., Delsingat P. // Nano Letters. 2006. Vol. 6. N 5. P. 937-941. https://doi.org/10.1021/n1052526t

[10] Yoo M.J., Fulton T.A., Hess H.F., Willett R.L., Dunkleberger L.N., Chichester R.J., Pfeiffer L.N., West K.W. // Science. 1997. Vol. 276. N 5312. P. 579-582. https://doi.org/10.1126/science.276.5312.579

[11] Li M., Tang H.X., Roukes M.L. // Nature Nanotechnol. 2007. Vol. 2. P. $114-120$. https://doi.org/10.1038/nnano.2006.208

[12] Cui X., Freitag M., Martel R., Brus L., Avouris P. // Nano Lett. 2003. Vol. 3. N 6. P. 783-787. https://doi.org/10.1021/n1034193a 
[13] Coffey D.C., Ginger D.C. // Nature Mater. 2006. Vol. 5. P. 735-740. https://dx.doi.org/10.1038/nmat 1712

[14] Borgani R., Forchheimer D., Bergqvist J., Thoren P.-A., Inganas O., Haviland D.B. // Appl. Phys. Lett. 2014. Vol. 105. N 14. P. 143113. https://doi.org/10.1063/1.4897966

[15] Maehashi K., Katsura T., Kerman K., Takamura Y., Matsumoto K., Tamiya E. // Analyt. Chem. 2007. Vol. 79. N 2. P. $782-787$. https://doi.org/10.1021/ac060830g

[16] Chen K.-I., Li B.-R., Chen Y.-T. // NanoToday. 2011. Vol. 6. N 2. P. 131-154. https://doi.org/10.1016/j.nantod.2011.02.001

[17] Kim D.-S., Jeong Y.-T., Park H.-J., Shin J.-K., Choi P., Lee J.H., Lim G. // Biosensors Bioelectron. 2004. Vol. 20. N 1. P. 69-74. https://doi.org/10.1016/j.bios.2004.01.025

[18] Yan R., Park J.-H., Choi Y., Heo C.-J., Yang S.-M., Lee L.P., Yang P. // Nature Nanotechnol. 2012. Vol. 7. P. 191-196. https://doi.org/10.1038/nnano.2011.226

[19] Qing Q., Jiang Z., Xu L., Gao R., Mai L., Lieber C.M. // Nature Nanotechnol. 2014. Vol. 9. P. 142-147. https://doi.org/10.1038/nnano.2013.273

[20] Presnova G., Presnov D., Krupenin V., Grigorenko V., Trifonov A., Andreeva I., Ignatenko O., Egorov A., Rubtsova M. // Biosensors Bioelectron. 2017. Vol. 88. P. 283-289. https://doi.org/10.1016/j.bios.2016.08.054

[21] Rubtsova M., Presnova G., Presnov D., Krupenin V., Grigorenko V., Egorov A. // Proced. Technol. 2017. Vol. 27. P. 234-235. https://doi.org/10.1016/j.protcy.2017.04.099

[22] Krupenin V.A., Presnov D.E., Zorin A.B., Niemeyer J. // Phys. B: Condens. Matter. 2000. Vol. 284-288. N 2. P. $1800-1801$. https://doi.org/10.1016/S0921-4526(99)02990-7

[23] Shorokhov V.V., Presnov D.E., Amitonov S.V., Pashkin Yu.A., Krupenin V.A. // Nanoscale. 2017. Vol. 9. N 2. P. 613-620. https://doi.org/10.1039/C6NR07258E

[24] Dagesyan S.A., Shorokhov V.V., Presnov D.E., Soldatov E.S., Trifonov A.S., Krupenin V.A. // Nanotechnology. 2017. Vol. 28. N 22. P. 225304. https://doi.org/10.1088/1361-6528/aa6dea

[25] Преснов Д.Е., Дагесян С.А., Божьев И.В., Шорохов В.В., Трифонов А.С., Шемухин А.А., Сапков И.В., Прохорова И.Г., Снигирев О.В., Крупенин В.А. // Вестн. Моск. ун-та. Сер. 3. Физ. астрон. 2019. N 2. С. 64-68. http://vmu.phys.msu.ru/file/2019/2/19-2-064.pdf [Presnov D.E., Dagesyan S.A., Bozhev I.V., Shorokhov V.V., Trifonov A.S., Shemukhin A.A., Sapkov I.V., Prokhorova I.G., Snigirev O.V., Krupenin V.A. // Moscow Univ. Phys. 2019. Vol. 74. N 2. P. $165-170$. https://doi.org/10.3103/S0027134919020164

[26] Stern J.E., Terris B.D., Mamin H.J., Rugar D. // Appl. Phys. Lett. 1988. Vol. 53. N 26. P. 2717-2719. https://doi.org/10.1063/1.100162

[27] Domansky K., Leng Y., Williams C.C. // Appl. Phys. Lett. 1993. Vol. 63. N 11. P. $1513-1515$. https://doi.org/10.1063/1.110759

[28] Salfi J., Savelyev I., Blumin M., Nair S.V., Ruda H.E. // Nature nanotechnol. 2010. Vol. 5. N 10. P. 737-741. https://doi.org/10.1038/nnano.2010.180

[29] Presnov D.E., Amitonov S.V., Krutitskii P.A., Kolybasova V.V., Devyatov I.A., Krupenin V.A., Soloviev I.I. // Beilstein J. Nanotechnol. 2013. Vol. 4. P. 330-335. https://doi.org/10.3762/bjnano.4.38
[30] Trifonov A.S., Presnov D.E., Bozhev I.V., Evplov D.A., Desmaris V., Krupenin V.A. // Ultramicroscopy. 2017. Vol. 179. P. 33-40. https://doi.org/10.1016/j.ultramic.2017.03.030

[31] Presnov D.E., Bozhev I.V., Miakonkikh A.V., Simakin S.G., Trifonov A.S., Krupenin V.A. // J. Appl. Phys. 2018. Vol. 123. N 5. P. 054503. https://doi.org/10.1063/1.5019250

[32] de Pablo P.J., Colchero J., Gómez-Herrero J., Baró A.M. // Appl. Phys. Lett. 1998. Vol. 73. N 22. P. 3300-3302. https://doi.org/10.1063/1.122751

[33] Автореф. канд. дис. Быков И.В. Развитие и автоматизация методов измерения рельефа и локальных свойств биологических объектов в атомно-силовой микроскопии. 01.04.01. M. 2010. https://search.rsl.ru/ru/record/01004651194

[34] Божьев И.В., Трифонов А.С., Преснов Д.Е., Дагесян С.А., Дорофеев А.А., Циняйкин И.И., Крупенин В.А. // Вестн. Моск. ун-та. Сер. 3. Физ. астрон. 2020. N 1. C. 42-48. [Bozhev I.V., Trifonov A.S., Presnov D.E., Dagesyan S.A., Dorofeev A.A., Tsiniaikin I.I., Krupenin V.A. // Moscow Univ. Phys. 2020. Vol. 1. N 1. P. 42-48.] http://vmu.phys.msu.ru/abstract/2020/1/20-1-042/

[35] Reimer L. in Scanning Electron Microscopy: Physics of Image Formation and Microanalysis. Springer. Berlin. Heidelberg, 1985. P. 53. https://doi.org/10.1007/978-3-662-13562-4 